\title{
Oral hyperpigmented macules observed during endoscopy intubation
}

\author{
Da Mi Kim', Gwang Ha Kim ${ }^{1,2}$, Moon-Bum Kim ${ }^{3}$ \\ ${ }^{1}$ Department of Internal Medicine, Pusan National University College of Medicine, Busan; ${ }^{2}$ Biomedical Research Institute, Pusan National University Hospital, \\ Busan; ${ }^{3}$ Department of Dermatology, Pusan National University College of Medicine, Busan, Korea
}

A 73-year-old woman visited our hospital for endoscopic resection of a gastric adenoma measuring approximately $1.2 \mathrm{~cm}$. She had no history of smoking or intake of medications such as tetracycline or chemotherapeutic agents that might be associated with mucocutaneous pigmentation. She had undergone a colonoscopy 2 years ago that revealed several tiny polyps, which were histopathologically diagnosed as low-grade tubular adenoma. Multiple hyperpigmented macules were observed on the tongue and buccal mucosa when introducing the endoscope into her oral cavity for gastric adenoma resection (Fig. 1A). Endoscopic submucosal dissection was successfully performed, and the final pathologic diagnosis was low-grade dysplasia. A detailed history after the procedure revealed that the dark macules had existed for 10 years on the inner aspect of her lower lip (Fig. 1B), and these macules had been consistently increasing in number. Dermoscopy of the labial lesions revealed a brownish reticular pattern along with granules (Fig. 1C). Longitudinal brownish-black streaks were also observed on the left second fingernail and right thumbnail (Fig. 1D). There was no itching or pain associated with the lesions, nor did she have any underlying disease or family history of skin pigmentation. The patient

\section{Received: October 30, 2020 Revised: December 11, 2020 \\ Accepted: December 13, 2020 \\ Correspondence: Gwang Ha Kim \\ Department of Internal Medicine, Pusan National University College of Medicine and Biomedical Research Institute, Pusan National University Hospital, 179 Gudeok-ro, Seo-gu, Busan 49241, Korea \\ E-mail: doc0224@pusan.ac.kr}

(a) This is an Open Access article distributed under the terms of the Creative Commons Attribution Non-Commercial License (http://creativecommons.org/licenses/by-nc/4.0/) which permits unrestricted non-commercial use, distribution, and reproduction in any medium, provided the original work is properly cited. was diagnosed with Laugier-Hunziker syndrome.

Laugier-Hunziker syndrome typically occurs during adulthood, and its exact etiology remains unknown. To date, evidence supporting an increased risk of malignancy is lacking. This syndrome occurs predominantly in middle-aged women. ${ }^{1}$ It is characterized by mucocutaneous pigmentatory lesions observed as lenticular melanotic macules on oral, labial, and acral areas. ${ }^{2}$ Longitudinal brownish-black streaks occur in approximately half of the patients. ${ }^{3}$ Histopathologically, melanin
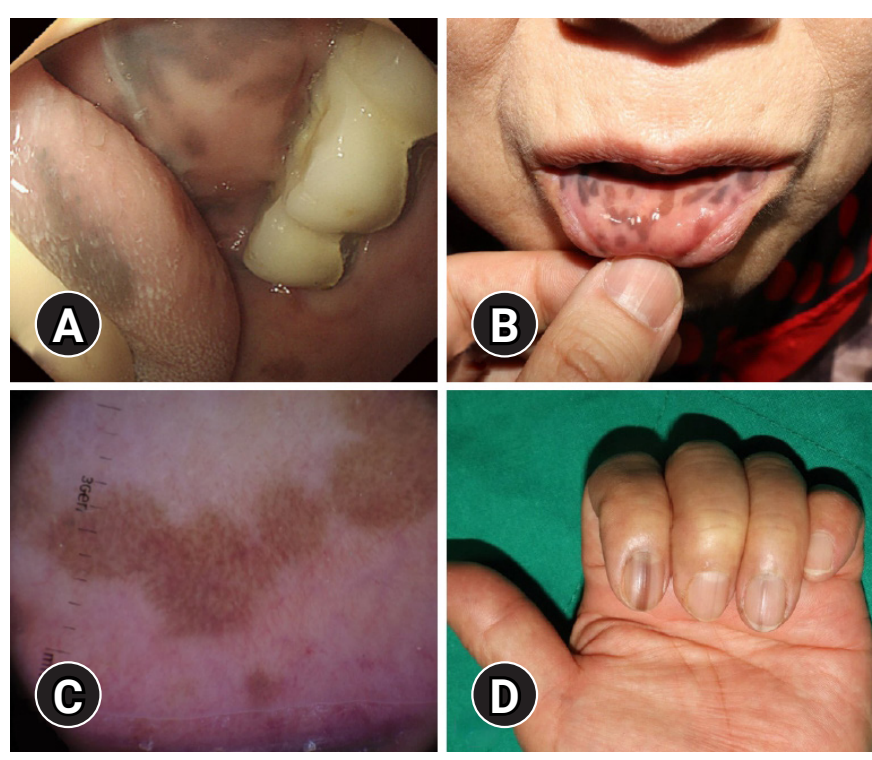

Fig. 1. (A) Multiple hyperpigmented macules observed on the tongue and buccal mucosa when introducing the endoscope into the oral cavity. (B) Dark macules on the inner aspect of the lower lip. (C) Dermoscopy of the labial lesions showing a brownish reticular pattern along with granules. (D) Longitudinal brownish-black streaks on the left second fingernail. 
accumulates in the cells of the basal layer of the dermis or epithelium, and there is an increase in the number of melanophages in the dermis or lamina propria. ${ }^{4}$ Generally, hyperpigmented macules do not disappear naturally; in contrast, they slowly increase with age. The diagnosis of this disease entity is mainly made clinically based on exclusion. ${ }^{2}$ Therefore, it is important to exclude other diseases such as Peutz-Jeghers syndrome. In Peutz-Jeghers syndrome, hyperpigmented macules of oral and acral areas usually appear in early life. In contrast, lesions in Laugier-Hunziker syndrome usually occur in adulthood. ${ }^{5}$ Peutz-Jeghers syndrome typically does not involve the tongue, palate, or fingernails, unlike Laugier-Hunziker syndrome. Furthermore, Peutz-Jeghers syndrome is associated with hamartomatous polyps of the gastrointestinal tract, whereas Laugier-Hunziker syndrome is not. ${ }^{5}$ Treatment is usually not required for Laugier-Hunziker syndrome, given the lack of associated systemic complications or malignant transformations. Our case suggests that detailed examination of the oral cavity when introducing the endoscope into the esophagus could provide clues for the diagnosis of rare dermatologic diseases.

\section{Conflicts of Interest}

The authors have no potential conflicts of interest.

\section{Funding}

None.

\section{Author Contributions}

Conceptualization: GHK; Data curation: DMK, MBK; Resources: GHK, MBK; Writing-original draft: DMK, GHK; Writing-review \& editing: DMK, GHK, MBK.

\section{ORCID}

Da Mi Kim Gwang Ha Kim https://orcid.org/0000-0002-2034-1887 https://orcid.org/0000-0001-9721-5734 Moon-Bum Kim https://orcid.org/0000-0003-4837-0214

\section{REFERENCES}

1. Nikitakis NG, Koumaki D. Laugier-Hunziker syndrome: case report and review of the literature. Oral Surg Oral Med Oral Pathol Oral Radiol 2013;116:e52-e58.

2. Duan N, Zhang YH, Wang WM, et al. Mystery behind labial and oral melanotic macules: clinical, dermoscopic and pathological aspects of Laugier-Hunziker syndrome. World J Clin Cases 2018;6:322-334.

3. Veraldi S, Cavicchini S, Benelli C, et al. Laugier-Hunziker syndrome: a clinical, histopathologic, and ultrastructural study of four cases and review of the literature. J Am Acad Dermatol 1991;25:632-636.

4. Zaki H, Sabharwal A, Kramer J, et al. Laugier-Hunziker syndrome presenting with metachronous melanoacanthomas. Head Neck Pathol 2019;13:257-263.

5. Cusick EH, Marghoob AA, Braun RP. Laugier-Hunziker syndrome: a case of asymptomatic mucosal and acral hyperpigmentation. Dermatol Pract Concept 2017;7:27-30. 